\title{
mRNA Expression Analysis of Tissue Sections and Single Cells
}

\author{
James Eberwine, ${ }^{1}$ Janet Estee Kacharmina, ${ }^{1}$ Christine Andrews, ${ }^{1}$ Kevin Miyashiro, ${ }^{1}$ Tracy Mclntosh, ${ }^{2}$ \\ Kevin Becker, ${ }^{4}$ Tanya Barrett,, ${ }^{4}$ Dave Hinkle, ${ }^{1,3}$ Gersham Dent, ${ }^{1}$ and Paolo Marciano ${ }^{2}$ \\ Departments of ${ }^{1}$ Pharmacology and Psychiatry, ${ }^{2}$ Neurosurgery, and ${ }^{3}$ Neurology, University of Pennsylvania Medical \\ Center, Philadelphia, Pennsylvania 19104, and ${ }^{4}$ DNA Array Unit, Gerontology Research Center, National Institute on \\ Aging, National Institutes of Health, Baltimore, Maryland 21224
}

Thirty years of literature have shown that changes in mRNA abundance provide insight into cellular functioning. Indeed, mRNA abundance measurements have been used to determine how effective particular drugs are in eliciting cellular responses, in monitoring behavioral responses, and in several other areas of neurobiology. Although investigation of individual mRNAs was informative, there are many thousands of mRNAs expressed in individual cells at varying abundances, the combination of which gives rise to cellular functioning. The advent of high-density cDNA arrays has greatly facilitated the ability to simultaneously examine the abundances of multiple mRNAs (Gress et al., 1992; Lockhart et al., 1996).

There are many areas warranting comment when discussing microarrays, only a few of which can be discussed in a minireview. One issue of importance is the relative merit in screening a macroarray versus a microarray. In general, a macroarray is a set of nucleic acids, such as cDNAs or oligonucleotides, that have been spotted onto a nylon membrane. Macroarrays are usually probed with radioactive probes. Alternatively, a microarray uses a glass slide as an immobile substrate to which cDNAs or oligonucleotides can be attached or directly synthesized. Microarrays are generally probed with fluorescently tagged probes. Although most investigators choose to use microarrays because of the range of signal detection, macroarrays have some distinct advantages. Most notably, macroarrays are cheaper and easier to make. Moreover, the use of radioactivity for signal detection is more sensitive than fluorescence-based detection. However, currently only one probe at a time can be screened on a macroarray, whereas a microarray uses two probes in a competitive hybridization. Consequently, it may be difficult to directly compare multiple macroarrays because of interarray variations. Nonetheless, given that more DNA can be bound to the filter than to glass, generally a stronger signal can be produced on a macroarray. The choice of array platform should be determined by the data requirements of the experiment.

Of particular note is the preparation of the probe for screening. The most popular method uses fluorescent cDNA probes made by reverse transcription of mRNA using fluorescently tagged nucleotides. This procedure generally requires between $500 \mathrm{ng}$ and 5 $\mu \mathrm{g}$ of starting mRNA. The amount of starting tissue required to produce this amount of mRNA translates to between 70 and 700 $\mu \mathrm{g}$ of wet tissue weight or between $10^{6}$ and $10^{7}$ cells. Clearly, with so many cells represented the ensuing microarray data must

Correspondence should be addressed to James Eberwine, Departments of Pharmacology and Psychiatry, University of Pennsylvania Medical Center, 36th and Hamilton Walk, Philadelphia, PA 19104. E-mail: eberwine@pharm.med.upenn.edu. Copyright (C) 2001 Society for Neuroscience $0270-6474 / 01 / 218310-05 \$ 15.00 / 0$ be viewed as a population result. Such data are critical to understanding the systems aspect of CNS responsiveness to modulation. However, if the experimental design requires more refined analysis, then this systems approach is inadequate.

Fortunately, the mRNA from a small amount of tissue, including a single cell, can be amplified to the levels required for an array probe. There are two generally used amplification procedures that can be used to generate a probe: PCR (Saiki et al., 1986) and the amplified antisense RNA (aRNA) procedure (VanGelder et al., 1990; Eberwine et al., 1992). The PCR procedure produces high levels of amplification, but any skewing of amplification efficiencies for particular mRNAs is exponentially amplified. The aRNA approach is a linear procedure in which skewing would be linearly amplified; consequently microarray results are more reflective of the initial mRNA abundances. In this review we will describe data generated using the aRNA approach.

\section{Array analysis of dendrites}

One adjunct of mRNA amplification and array analyses is the capability to distinguish the complement of mRNAs present in different neuronal subdomains. Previous studies have revealed that a complex subset of mRNAs are present within the dendritic subdomain, where their local translation may aid in modulating plastic events at neuronal synapses (Miyashiro et al., 1994). It was first proposed by Spirin (1969) that cellular mRNAs are complexed with specific proteins to form aggregates more commonly referred to as messenger ribonucleoprotein particles or informosomes. RNAs in the membrane-bound ribosome fraction are associated with higher rates of translation. Several reports indicate that functional differences may exist between the free and bound ribosomes, with each retaining different kinds of mRNAs coding for specific proteins. In an effort to analyze the functional correlation of free versus membrane-bound ribosome fractions and their corresponding RNA content, we have begun experiments aimed at isolating polysome fractions of isolated dendrites. Free and membrane-bound ribosome fractions are isolated by traditional discontinuous sucrose density gradient centrifugation techniques (Murthy, 1972). The mRNA contents of the free and membrane-bound polysome fractions were reverse transcribed with an oligo-dT-T7 RNA polymerase promoter. The contents were taken through the aRNA procedure and applied to macroarrays and microarrays. As initial proof of mRNA content, we combined both free and membrane-bound polysome fractions of whole hippocampal cells in culture, extracted their mRNA complement, and applied the resulting material to Research Genetics (Huntsville, AL) microarrays (Fig. 1A). These experiments may provide insight into determining the functional differences between these ribosomal fractions. 

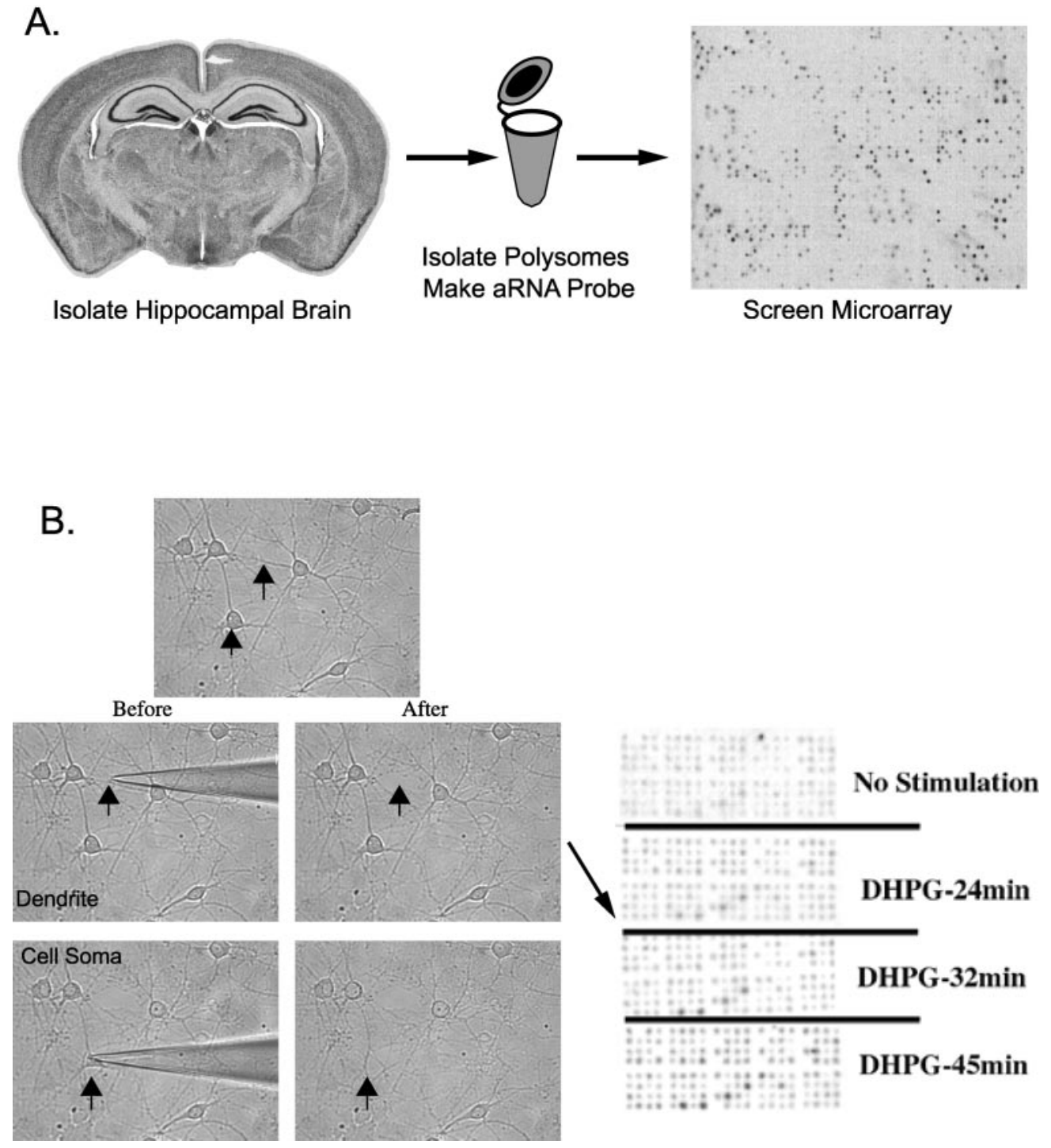

Figure 1. Use of DNA arrays to examine dendrite molecular biology. $A$ schematizes the harvesting of hippocampal tissue for use in isolation of polysomes and the array result. $B$ shows the harvesting of dendrites and a cell soma (indicated by arrows) using a microelectrode as a scalpel. After DHPG treatment, the dendrites are microdissected and an aRNA probe is synthesized; this probe was used to probe a homemade macroarray shown in $B$.

Because mRNAs can be localized to dendrites, it is of interest to determine the rates of transport for different species of dendritic mRNAs. Similar rates would suggest that a similar mechanism mediates the transport of the co-regulated mRNAs. In addition, numerous lines of evidence demonstrate that local dendritic protein synthesis does occur. Polyribosomes have been found in dendrites and close to the base of dendritic spines (Steward, 1997). Immunohistochemistry has been used to detect several components of the translational machinery (Tiedge and Brosius, 1996; Gardiol et al., 1999). Epitope-tagged mRNAs transfected into isolated neuronal dendrites were locally translated (Crino and Eberwine, 1996; Kacharmina et al., 2000). Evidence that dendrites are translationally competent adds importance to identifying which mRNAs are transported into dendrites.

Microarray analysis coupled with aRNA has been used to screen for genes that are enriched in neuronal dendrites in response to various stimuli. For example, $(R S)$-3,5-dihydroxyphenylglycine (DHPG), a metabotrobic glutamate receptor agonist, and neurotropic factors BDNF or NT3 are all reagents that modulate protein translation in dendrites (Weiler and Greenough, 1993; Kang and Schuman, 1996) and have been used to stimulate dendritic translation in rat hippocampal cultures. We treated primary rat hippocampal neuronal cultures with DHPG and severed the dendrites at different times after stimulation (Eberwine et al., 2001). A patch pipette was used to harvest individual dendrites from these cultured neurons, as can be seen in Figure $1 B$, top. Individual cell soma can also be isolated from the same cultures, as shown in Figure $1 B$, bottom. ${ }^{33} \mathrm{P}$-labeled aRNA was prepared from the harvested dendritic mRNA and was used to screen macroarrays (Fig. $1 B$, side). These data show that the abundances of a few mRNAs are increased in dendrites as a result of DHPG stimulation, but that the rate of mRNA transport varies for the different mRNAs.

\section{Array analysis from fixed tissue sections}

The analysis of mRNA populations from selected brain regions has been performed previously by Sandberg et al. (2000) using pieces of dissected fresh mouse brain tissue. However oftentimes only small amounts of tissue are available, and usually it is fixed rather than fresh tissue. To accommodate this, the aRNA procedure has been modified. mRNA can be transcribed in situ into cDNA on the tissue section (Tecott et al., 1988) using an oligodT-T7 primer (Crino et al., 1996). This primer will anneal to the poly-A + mRNA and will serve as a primer for cDNA synthesis in situ. The harvesting of cDNA from the cell of interest can be 
Figure 2. Microdissection of the mouse amygdala and macroarray analysis of subregions. Sections through the mouse brain were stained with cresyl violet to visualize subregions of the amygdala for dissection. Neuroarray hybridization results are shown for the basolateral and central nuclei. Arrows highlight cDNAs for which mRNA abundances differ between basolateral and control nuclei.

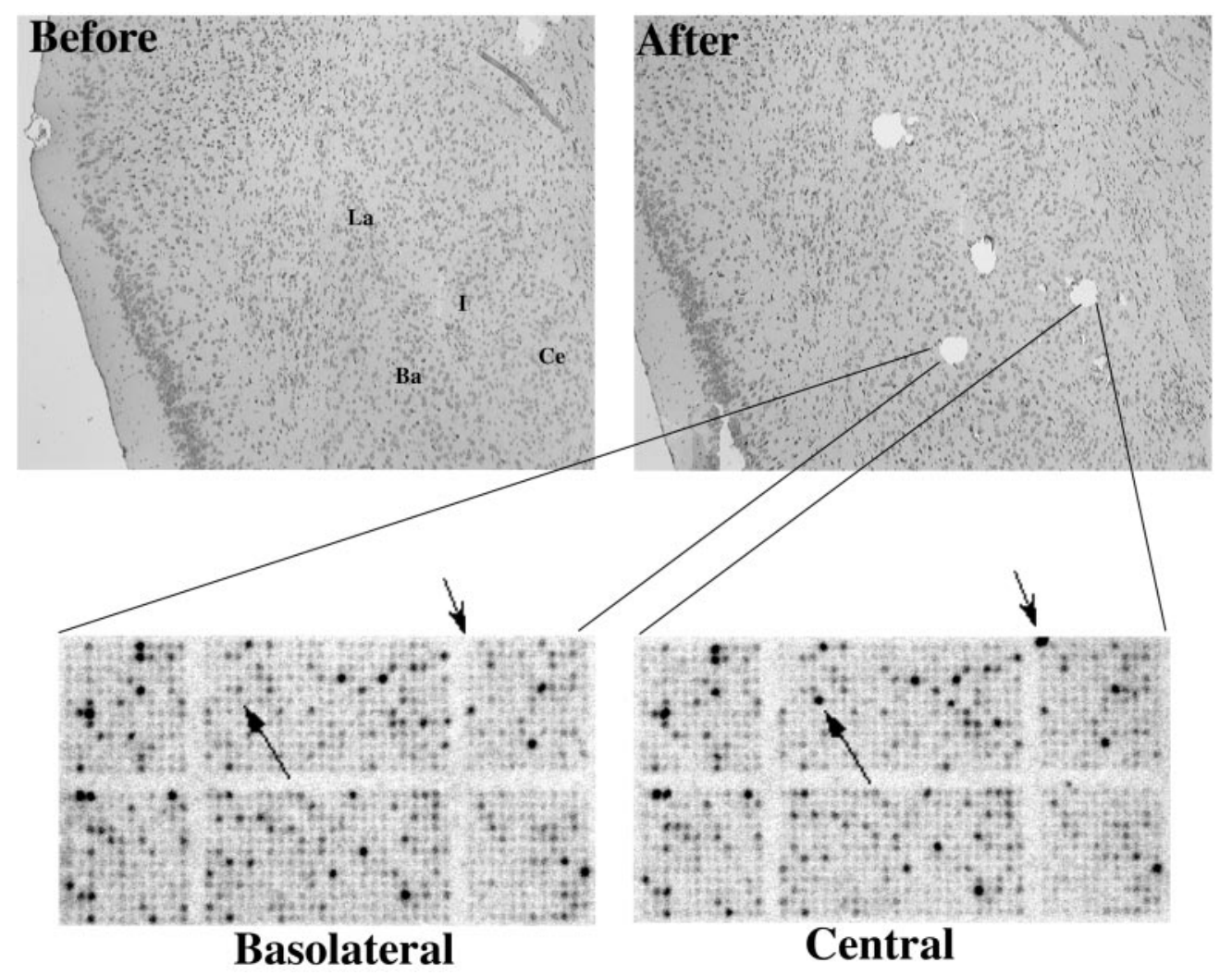

directed by the immunostaining. aRNA amplification of this cDNA is used to screen microarrays. Such an analysis is particularly critical in the study of human diseases in which the most easily obtainable tissue is from fixed pathological specimens (Crino et al., 1996; Chow et al., 1998). The cDNA harvesting can be accomplished using a patch electrode or laser capture microdissection (Luo et al., 1999). The ability to examine the mRNA complement of fixed tissues with DNA microarrays helps abate the gap between molecular and systems neuroscience.

The molecular mechanisms underlying mammalian behaviors have been difficult to examine, primarily because of the large number of genes that coordinate a behavioral phenotype and the heterogeneous cell composition of the CNS. Because it is known that behavior results from coordinated changes in multiple genes, microarrays offer the ability to simultaneously examine several mRNAs in a region relevant to the behavioral task. For example, limbic forebrain structures, specifically the amygdaloid complex, are part of neural pathways mediating multiple behaviors (Gallagher et al., 1990). Neuroanatomical studies have determined that the amygdala is not a unitary region; instead it is a collection of several interconnected subnuclei (Pitkanen et al., 1997). To better understand the intra-amygdaloid molecular interactions, we are using microarray analysis to examine gene expression profiles of individual amygdala subregions in response to various pharmacological challenges. Initially, cresyl violet is used to stain fixed paraffin-embedded sections to visualize amygdala subregions (Fig. 2). After in situ transcription, the cresyl violet staining is used to direct the microdissection of amygdala subnuclei. Subsequent to harvesting, the cDNA was used to generate an aRNA probe. The expression profile of a control basolateral nucleus visualized on a neuroarray platform (National Institute of Aging) is presented in Figure 2, and the hybridization intensities of the different cDNAs are similar to that of the central nucleus, with a few notable exceptions (Fig. 2, arrows). Such large differences in abundance are the exception rather than the rule. It may be possible to use the promoter of genes encoding regionally restricted mRNAs to drive transgenic expression in that selected amygdala region.

Sometimes it is desirable to sort through the cellular heterogeneity and perform cell-type-specific molecular analysis. Cells can be often be differentiated into subclasses based on the expression of multiple protein antigens. This is true for cells that die after a cellular insult such as traumatic brain injury (TBI) (O'Dell et al., 2000). Studies from our laboratory are currently using DNA microarrays to assess differential gene expression in selective mouse hippocampal neurons after TBI. After TBI, the apoptotic cell death pathway is activated, resulting in the death of cells in subregions of the hippocampus. We have determined the expression profile of active caspase-3-immunoreactive and terminal deoxynucleotidyl transferase-mediated biotinylated UTP nick end labeling (TUNEL)-positive neurons (Fig. $3 A$ ). Examination of the injured neuronal cell types (caspase negative, TUNEL negative; caspase positive, TUNEL negative; and caspase positive, TUNEL positive) should highlight those changes in gene expression that are time-dependent during the apoptotic process (Fig. 3B,C). Figure $3 C$ shows the scattergram of data collected from a microarray analysis of injured versus uninjured CA3 neurons. The red lines on the scattergram highlight twofold differences in mRNA abundance. These data may prove useful in the design of TBI-directed therapeutics.

\section{Cautions associated with DNA array use}

Important areas of genomic microarray analysis that have not been detailed in this review include the necessity of performing multiple repeats and additional secondary screens of array positives. Simply put, the more arrays screened, the better the statis- 


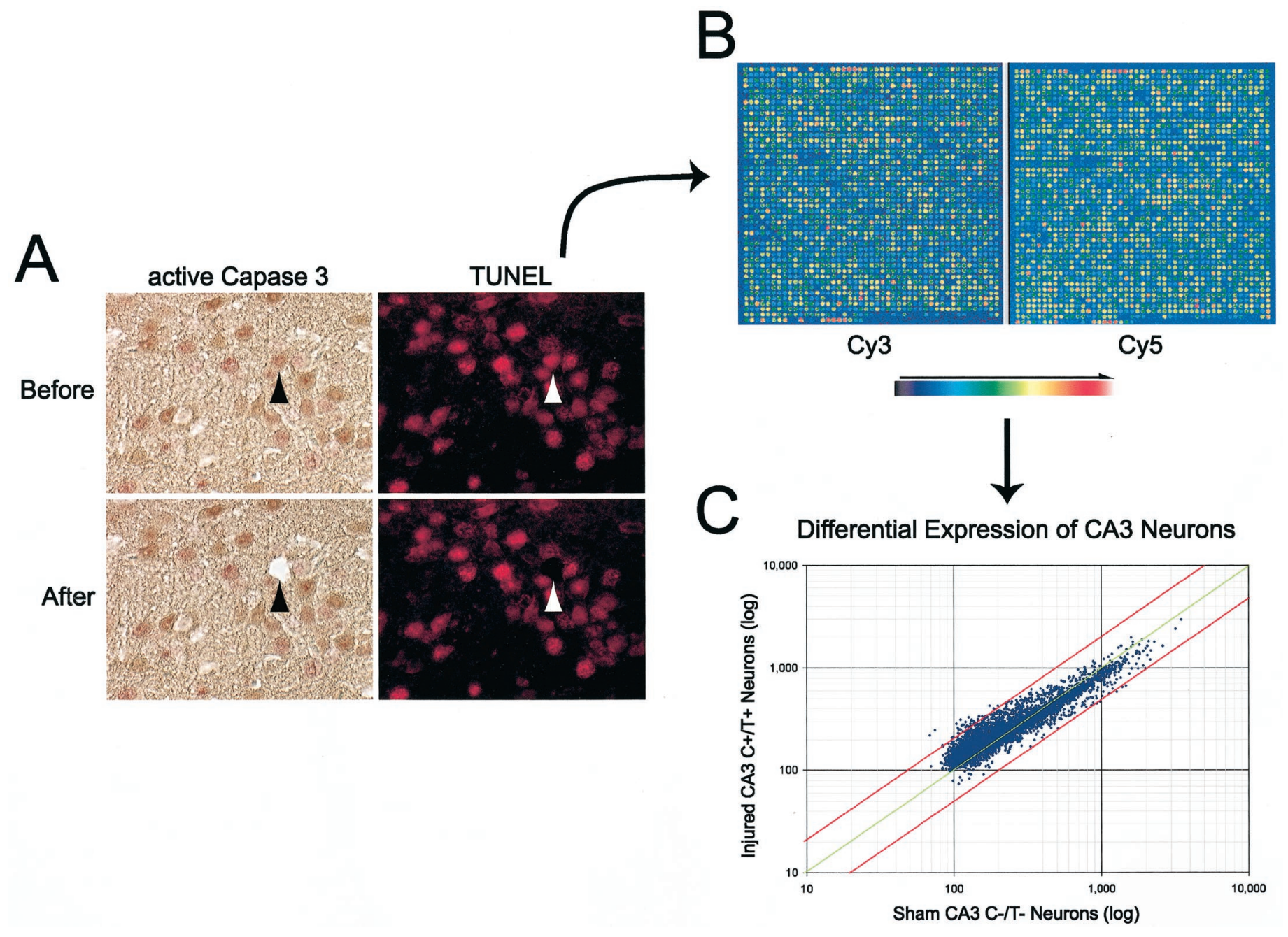

Figure 3. mRNA expression analysis of dying cells in traumatic brain injury. The arrows in $A$ show a cell of the CA3 region of the hippocampus that is caspase-3-positive and TUNEL-positive after TBI. aRNA probes were fluorescently labeled and applied to an Incyte (Palo Alto, CA) Gem microarray, a portion of which is shown in $B$. The scattergram of these array results is shown in $C$.

tical evaluation of differences in gene expression. There is certainly a cost constraint to performing multiple array analyses, but three arrays should be run at minimum and used to determine differential gene expression between two mRNA samples. In addition, secondary screens should be performed to confirm at least a subset of the microarray data. The type of secondary screen will vary depending on experimental details but common types used include Northern blot analysis, in situ hybridization, secondary subarrays, and real-time PCR. Another area that has been neglected in this review is bioinformatic analysis of the generated datasets. Although there are many useful prepackaged programs for data analysis, a bioinformatics specialist should be consulted during the early stages of experimental design and data analysis to ensure that experimental details are adequately controlled for.

Expression profiling provides a wealth of data concerning mRNA abundance differences between experimental samples; however, these differences are not always reflective of functional protein levels. The most desirable cellular analysis would be one that combines both mRNA and protein analysis. Although the field of proteomics is in its infancy, various groups (Ekstrom et al., 2001; Zhang et al., 2001) are currently developing methods that permit analysis of multiple proteins from tissue samples, including single cells. The marriage of genomics and proteomics promises to help in answering many of the questions that have captivated us for centuries.

\section{REFERENCES}

Chow N, Cox C, Callahan L, Weimer J, Guo L, Coleman P (1998) Expression profiles of multiple genes in single neurons of Alzheimer's disease. Proc Natl Acad Sci USA 95:9620-9625.

Crino P, Eberwine J (1996) Molecular characterization of the dendritic growth cone: regulated mRNA transport and local protein synthesis. Neuron 17:1173-1187.

Crino P, Dichter M, Trojanowski J, Eberwine J (1996) Single cell molecular pathology: an analysis of tuberous sclerosis. Proc Natl Acad Sci USA 93:14152-14157.

Eberwine J, Yeh H, Miyashiro K, Cao Y, Nair S, Finnell R, Zettel M, Coleman P (1992) Analysis of gene expression in single live neurons. Proc Natl Acad Sci USA 89:3010-3014.

Eberwine J, Miyashiro K, Kacharmina J, Job C (2001) Local translations of classes of mRNAs that are targeted to neuronal dendrites. Proc Natl Acad Sci USA 98:7080-7085.

Ekstrom S, Ericsson D, Onnerfjord P, Bengtsson M, Nilsson J, MarkoVarga G, Laurell T (2001) Signal amplification using "spot-on-a-chip" technology for the identification of proteins via MALDI-TOF MS Anal Chem 73:214-219.

Gallagher M, Graham P, Holland P (1990) The amygdala central nucleus and appetitive Pavlovian conditioning: lesions impair one class of conditioned behavior. J Neurosci 10:1906-1911. 
Gardiol A, Racca C, Triller A (1999) Dendritic and postsynaptic protein synthetic machinery. J Neurosci 19:168-179.

Gress T, Hoheisel J, Lennon G, Zehetner G, Lehrach H (1992) Hybridization fingerprinting of high-density cDNA-library arrays with cDNA pools derived from whole tissues. Mamm Genome 3:609-619.

Kacharmina J, Job C, Crino P, Eberwine J (2000) Stimulation of glutamate receptor protein synthesis and membrane insertion within isolated neuronal dendrites. Proc Natl Acad Sci USA 97:11545-11550.

Kang H, Schuman E (1996) A requirement for local protein synthesis in neurotrophin-induced hippocampal synaptic plasticity. Science 273:1402-1406.

Lockhart D, Dong H, Byrne M, Follettie M, Gallo M, Chee M, Mittmann M, Wang C, Kobayashi M, Horton H, Brown E (1996) Expression monitoring by hybridization to high-density oligonucleotide arrays. Nat Biotechnol 14:1675-1680.

Luo L, Salunga R, Guo H, Bittner A, Joy K, Galindo J, Xiao H, Rogers K, Wan J, Jackson M, Erlander M (1999) Gene expression profiles of laser-captured adjacent neuronal subtypes. Nat Med 5:117-122.

Miyashiro K, Dichter M, Eberwine J (1994) On the nature and distribution of mRNAs in hippocampal neurites: implications for neuronal functioning. Proc Natl Acad Sci USA 91:10800-10804.

Murthy MR (1972) Free and membrane-bound ribosomes of rat cerebral corts. Protein synthesis in vivo and in vitro. J Biol Chem 247:1036-1043.

O'Dell D, Raghupathi R, Crino P, Eberwine J, McIntosh T (2000) Traumatic brain injury alters the molecular fingerprint of TUNELpositive cortical neurons in vivo: a single-cell analysis. J Neurosci 20:4821-4828
Pitkanen A, Savander V, LeDoux J (1997) Organization of intraamygdaloid circuitries in the rat: an emerging framework for understanding functions of the amygdala. Trends Neurosci 20:517-523.

Saiki R, Bugawan T, Horn G, Mullis K, Erlich H (1986) Analysis of enzymatically amplified $\beta$-globin and HLA-DQ $\alpha$ DNA with allelespecific oligonucleotide probes. Nature 324:163-163.

Sandberg R, Yasuda R, Pankratz D, Carter T, Del Rio J, Wodicka L, Mayford M, Lockhart D, Barlow C (2000) Regional and strain-specific gene expression mapping in the adult mouse brain. Proc Natl Acad Sci USA 97:11038-11043.

Spirin AS (1969) The second Sir Hans Krebs lecture: Informasomes. Eur J Biochem 10:20-35.

Steward O (1997) mRNA localization in neurons: a multipurpose mechanism? Neuron 18:9-12.

Tecott L, Barchas J, Eberwine J (1988) In situ transcription: specific synthesis of cDNA in fixed tissue sections. Science 240:1661-1664.

Tiedge H, Brosius J (1996) Translational machinery in dendrites of hippocampal neurons in culture. J Neurosci 16:7171-7181.

VanGelder R, vonZastrow M, Yool A, Dement W, Barchas J, Eberwine J (1990) Amplified RNA (aRNA) synthesized from limited quantities of heterogeneous cDNA. Proc Natl Acad Sci USA 87:1663-1667.

Weiler IJ, Greenough W (1993) Metabotropic glutamate receptors trigger postsynaptic protein synthesis. Proc Natl Acad Sci USA 90:7168-7171.

Zhang H, Kacharmina J, Miyashiro K, Greene M, Eberwine J (2001) Protein quantification from complex protein mixtures using a novel proteomics methodology with single cell resolution. Proc Natl Acad Sci USA 98:5497-5502. 